\title{
Integrating Process Mapping Into The AIS Students' Toolset
}

Marianne Bradford, (Email: bradford@bryant.edu), Bryant College

Doug Roberts, (Email: froberts@utk.edu), Kansas State University

Gordon Stroupe (Email: stroupe_Gordon@emc.com), EMC Corporation

Introduction

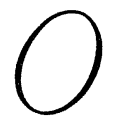

ver the past decade, the impact of technology on accounting practice has been profound. The professional landscape is rapidly changing, and today's accounting students have more career opportunities than ever before. While many accounting majors are still choosing the traditional routes of public or corporate accounting, others are tailoring their education toward management or systems consulting. As AIS educators it is imperative that we maintain currency in our curriculum for both the traditional majors and those students seeking a consulting career path.

Although the AIS curriculum is applicable to both types of accounting majors, certain content is especially pertinent to those students interested in consulting. One subject that consultants draw upon extensively is systems documentation. Current AIS curriculum focuses on analysis, design, and documentation of systems from an internal control and data flow perspective, and students learn to use traditional accounting documentation tools. These techniques include document and systems flowcharts and data flow diagrams. ${ }^{1}$ While these tools may be sufficient for their intended purposes, they are not the primary documentation techniques used in the consulting profession. The objective of this paper is to advocate the inclusion of a popular management consulting documentation technique, process mapping, into the accounting curriculum. Process maps are used to better understand a business entity's current business processes and communicate these in a non-technical way. Additionally, process mapping can provide an initial foundation to successful process management.

\section{Picture Your Business}

Business process is a collection of activities that takes one or more kinds of input and creates an output that is of value to the customer (Hammer and Champy, 1993). One of the primary tasks facing consultants today is reengineering of these business processes for their clients. This revolution has rapidly spread across the country as companies pursue promises of improved quality, service, productivity, and profitability (Boehringer and King, 2000). Consultants need to gain knowledge of their clients' business in an effort to improve, or reengineer, these processes. They are often expected to come up to speed quickly on the condition of the organization by mapping the current ("as is") state of various processes, which then will be used as a spring board to recommend future ("could be") state processes (oftentimes leveraging industry best practices) (Boehringer and King, 2000). A high level understanding of interdependencies and integration points gives the consultant a foundation from which to begin a more

Readers with comments or questions are encouraged to contact the authors via email.

\footnotetext{
${ }^{1}$ Document flowcharts show the flow of documents within a system. Systems flowcharts document the control points within a process. Data flow diagrams demonstrate the flow and disposition of data within organizations. Other documentation techniques included in systems textbooks are pseudocode, program flowcharts, and decision tables.
} 
detailed analysis. The understanding of business processes requires the use of documentation techniques other than the ones in an accountant's traditional toolset.

Understanding business processes is also key to performing consulting services involving enterprise wide systems. Currently, services related to enterprise systems generate one third to one half of total consulting revenue at national professional services firms (Public Accounting Report, 1998). Enterprise systems force organizations to consider how different departments interact with each other and how the process of doing business is accomplished throughout their firm. These packages alternatively known as enterprise resource planning (ERP) systems, use process maps to illustrate the standard software configuration. The configuration is typically modeled after generic business processes that in many cases may not support the adopting organization. Consultants use these process maps to identify differences in the ERP system's process flows and the organization's process flows in an effort to propose adjustments to the corporate process or configuration of the software. Only through the use of business process documentation can this be made feasible.

In process mapping, the level of detail can be from a broad organizational process perspective down to a micro-detail approach of the smallest unit of work. The focus is on process design and process management, key components to a successful AIS. And, because process maps are visual, anyone in an organization should be able to decipher them no matter what their educational background.

Among the various process-mapping techniques used, a cross-functional process map (CFPM) is especially valuable. ${ }^{2}$ This type of map shows the steps and functional units involved in the completion of a specific process, thereby documenting the "what" and "who". CFPMs provide an in-depth understanding of the interactions among the various functions involved with each process and provide management with a vision of the organization they may never have been able to picture otherwise.

Both existing business processes and reengineered processes must be easily communicated to people at all levels of the organization, and CFPMs present this information clearly and concisely. Furthermore, CFPMs present information about a business process in its organizational context. In other words, with one diagram a user can see all the steps (or events) involved in a business process, the organizational function that performs the steps, the dependencies in the process, and the order in which the steps are generally performed. The user can also see the sequential and concurrent nature of activities and the decision points. Unlike data flow diagrams, CFPMs take into consideration organizational units (process owners) and characterize how information moves throughout an organization as business is performed. Specific hardware or data stores are not necessarily represented in a CFPM unless they are considered to be an essential entity in a given business process.

\section{The Mechanics}

When a CFPM is created, the name of the business process is presented at the top of the map, and the map is numbered for uniqueness (Figure 1). The process map starts with a 'trigger' event, and each successive action is noted clearly and concisely. Process owners are delineated with either horizontal or vertical functional bands (often referred to as swim-lanes). ${ }^{3}$

To maintain consistency, these bands retain the same owner throughout the map. Tasks, or lower level sub-processes, that the functional owner is responsible for are presented in the appropriate

${ }^{2}$ CFPM is one of several different process mapping techniques. Other process mapping techniques

include assembly flowcharts, process flowcharts, and relationship maps (Adams, 2000).

${ }^{3}$ CFPMs may be drawn with process owner columns appearing horizontally or vertically. 
lane using numbered rectangles. The numbered rectangles are children tasks, or sub-processes, of the parent process and are numbered in succession.

FIGURE 1

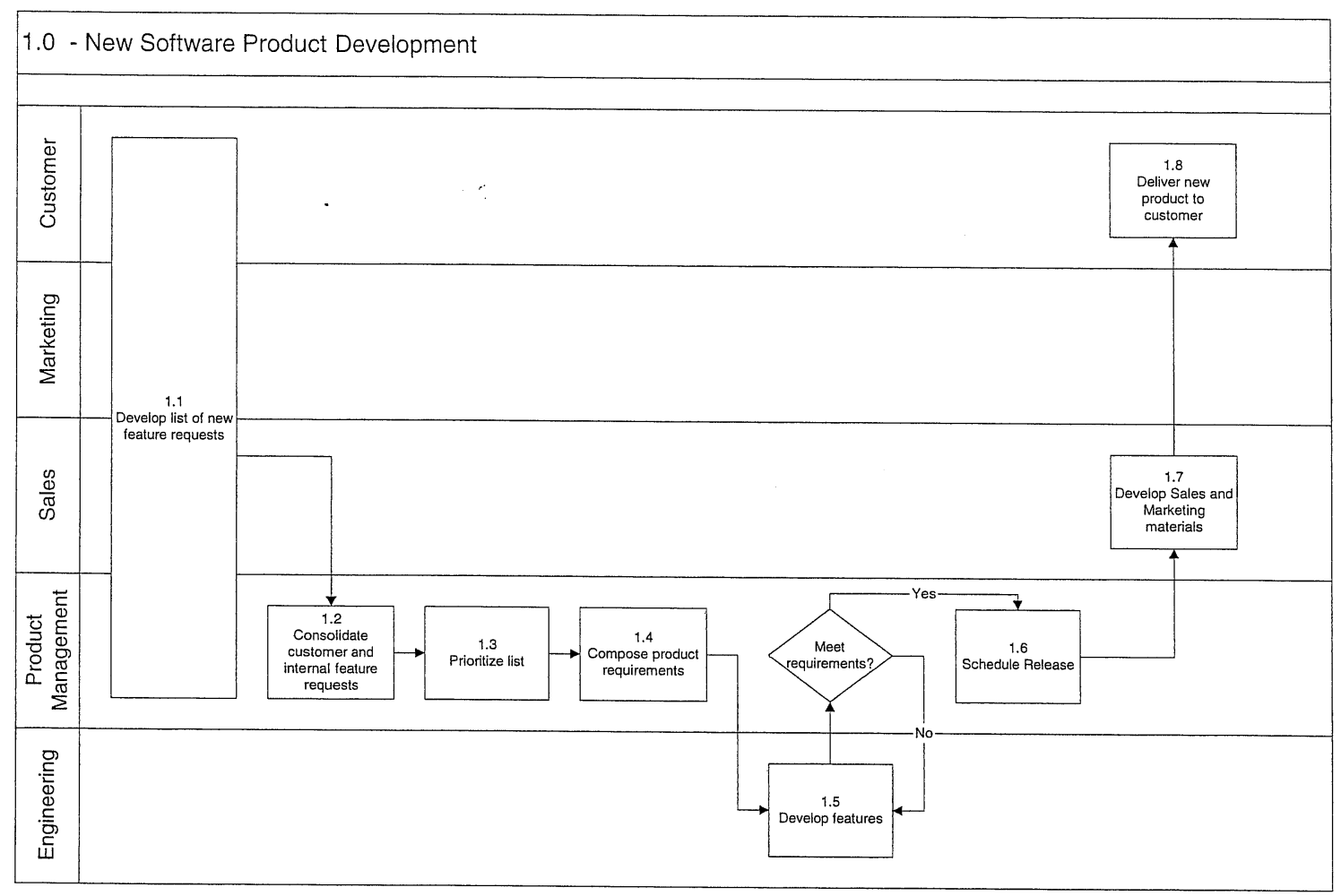

Some sub-processes are actually performed by multiple functional participants, such as subprocess 1.1 (Develop list of new feature requests). Creating one sub-process box across several swimlanes represents these sub-processes. Sub-processes that traverse functional boundaries may be performed in unique ways depending upon the organizational unit performing the task. In these cases, another process map can be used that accurately reflects the steps that each functional owner performs in completing the process (Figure 2).

Detailed task instructions may accompany the map and use the process numbers as a reference. For example, sub-process 1.1 (Develop list of new feature requests) could be accompanied by a detailed document that clearly articulates the steps and specific instructions used by the Marketing department to perform this sub-process (Figure 3). These detailed step-by-step instructions also include what department or person "owns" the process, the inputs and outputs of the process, and any dependencies of the process. Not only do the instructions thoroughly document the system, but they can also be used as a foundation for training materials. 


\section{Detailed documentation for Sub Process 1.1: Marketing}

Date: 25 May 2001

Author: J. Doe

Process Owner: Marketing

Customer: Product Management

\begin{tabular}{|l|l|}
\hline Step & Instructions \\
\hline 1 & $\begin{array}{l}\text { Organize focus groups with balanced technical and non-technical users to drive } \\
\text { new product features. Ensure that all participants complete the Product } \\
\text { Usabilïty Survey and receive compensation for their time. }\end{array}$ \\
\hline 2 & $\begin{array}{l}\text { Interview all participants individually and as a group to derive additional } \\
\text { features from the sessions. }\end{array}$ \\
\hline 3 & $\begin{array}{l}\text { Compare requested features with the most recent Product Roadmap found on the } \\
\text { Product Management intranet site. }\end{array}$ \\
\hline 4 & $\begin{array}{l}\text { Clearly and accurately document all feature requests (include a video taped copy } \\
\text { of each interview if possible). }\end{array}$ \\
\hline $\begin{array}{l}\text { Dependencies: None } \\
\text { Inputs: None } \\
\text { Outputs: Formal, documented, new feature requests delivered to Product Management }\end{array}$
\end{tabular}

FIGURE 2

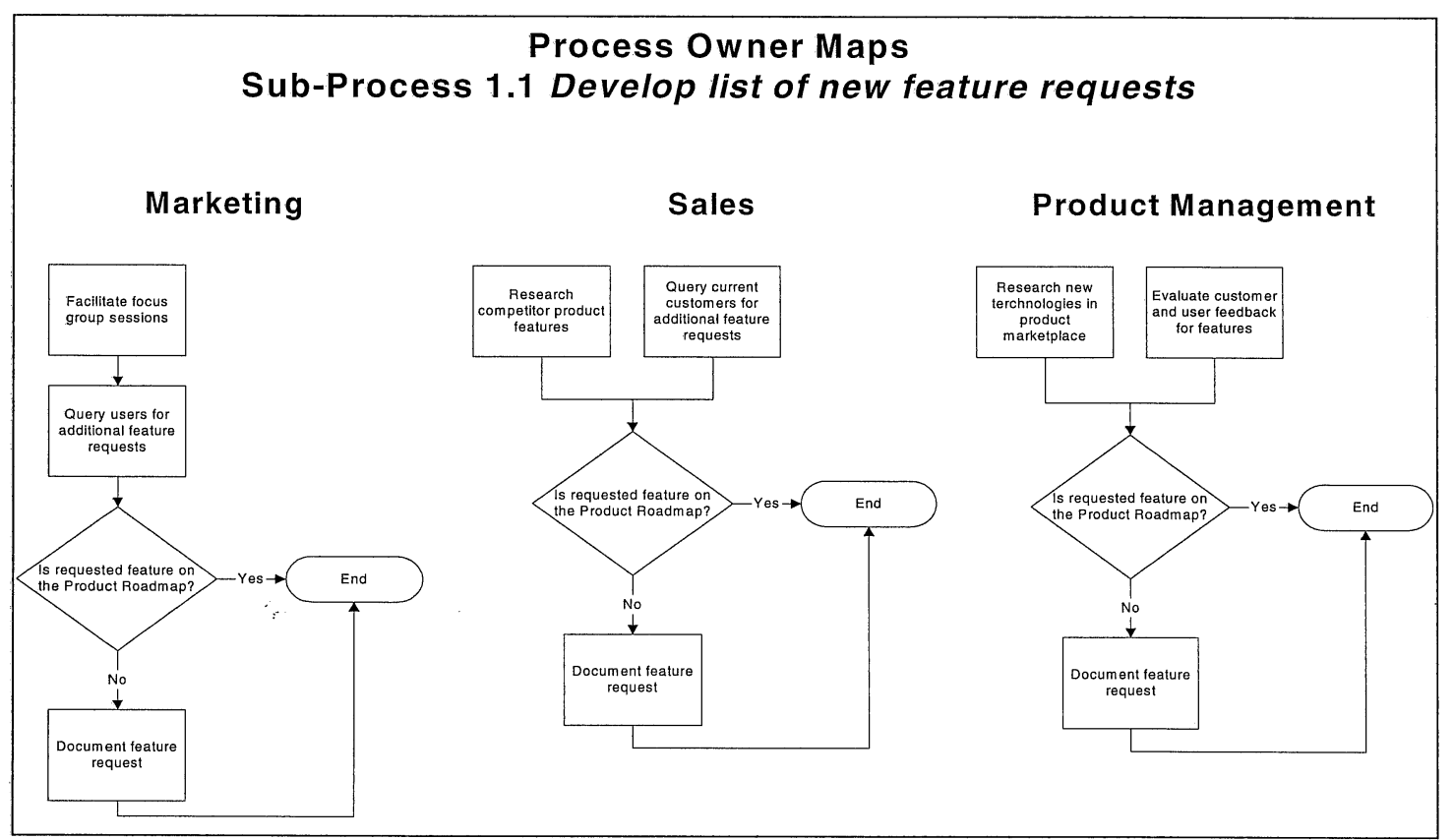

FIGURE 3 
Some sub-processes are in fact sophisticated cross-functional processes and require a subsequent map detailing their cross-functional nature. An example of a sub-process merits a second level process map is sub-process 1.4 (Compose product requirements). Figure 4 details the steps involved in performing a particular business activity.

\section{FIGURE 4}

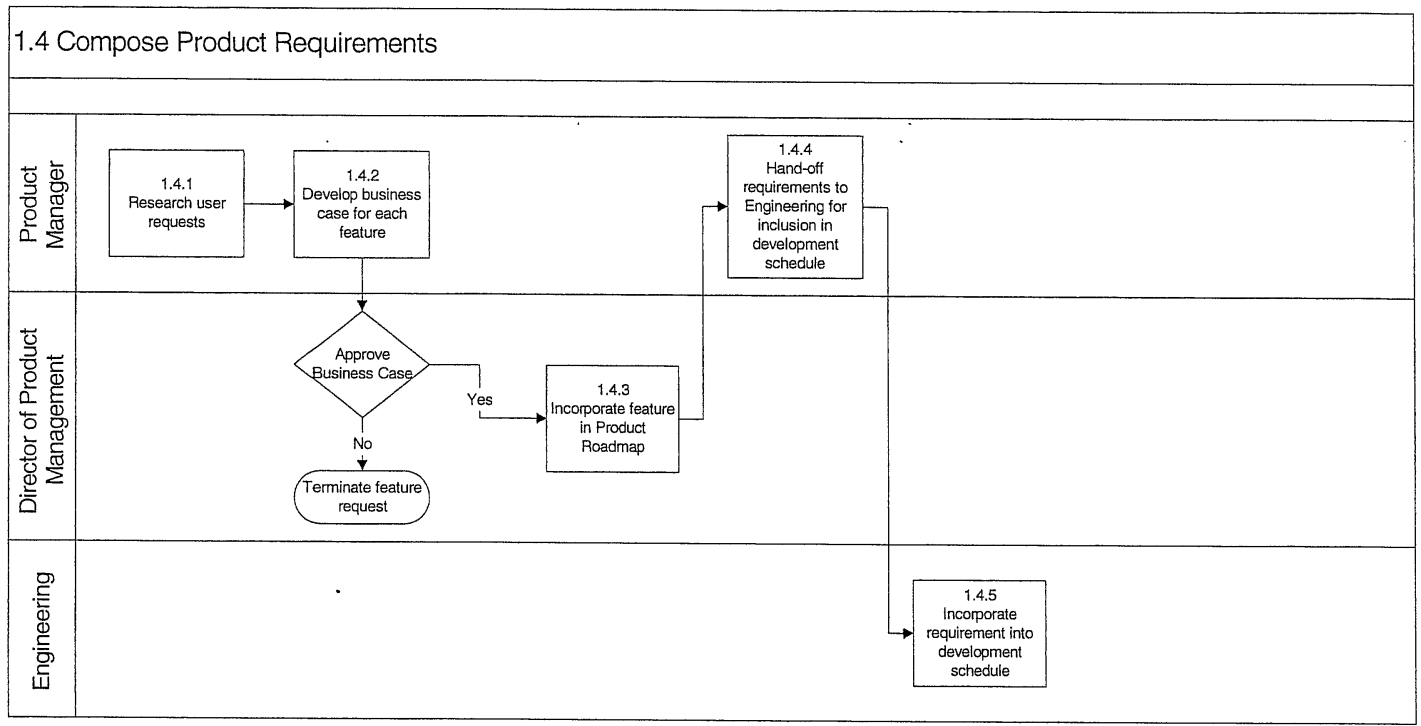

It is often useful to map business processes at a high level and then drill down to successive lower levels as we have demonstrated using first the main process of New Software Product Development and the sub-process Compose Product Requirements. This enables consultants to uncover flaws or inefficiencies in processes. For instance, at a high level, most manufacturers perform exactly the same tasks: (1) They buy things. (2) They add value to those things. (3) They sell the new things. However, as consultants "peel away at the layers of the onion" it becomes clearer and clearer where problems are and where improvements can be made. Many times a first or second level map is sufficient to locate problem areas.

CFPMs portray time in a relative sense to the reader. For example, using horizontal swim-lanes, time is represented from left to right and is not bound to any particular scale (i.e., hours or days). The first sub-process, 1.1 (Develop list of new feature requests), is performed prior to sub-process 1.2 (Consolidate customer and internal feature requests), but there is no explicit time scale in the map that gives the user a sense of how long a particular process (or series of processes) normally takes. ${ }^{4}$ Connectors between tasks or sub-processes represent dependencies and can be presented as optional using dotted lines. Decision points are represented using the traditional diamond shape and can be chained together to represent multiple decision points.

\footnotetext{
${ }^{4}$ Time is often communicated in the detailed description of the process that accompanies the map. For instance, in the header of the detailed description a placeholder for Estimated Time to Complete can be incorporated. The purpose of the map is to communicate interdependent relationships within an organization, not necessarily to record job and task measurements.
} 
Process mapping symbols can take the form of boxes, arrows and decision diamonds, or the toolset can be broadened, depending on the environment being documented and the task detail. In a manufacturing environment, the flow time, or distance a part travels, is of critical importance. Symbols can be used to denote delays, such as queue time or setup time, inspection of parts, rejects of parts and storage of parts. Using these symbols to document the flow of materials from raw material to shipping can aid in pinpointing excessive delays and handoffs and inefficient movement of goods. Isolating these problems through the use of process mapping can lead to dramatic improvements in flow rates. This simple, yet effective presentation format is one of the strengths of the CFPM because it can illustrate complicated organizational interdependencies easily.

\section{Applying Process Maps}

In the case of a systems design project, once the consultant has documented a new process to the appropriate level and included the process maps in a functional design document, a systems architect or systems analyst can then take the functional design and begin developing technical design documents, which in many cases include a series of data flow diagrams and database schemas. From there, software engineers develop the software that will be used to support the newly re-engineered process.

Learning to use process-mapping techniques is an invaluable skill for the businessperson in the field. Poorly designed processes, redundant workflow, and inadequate controls can be easily identified in a properly documented CFPM, and a redesigned process can be developed and presented next to the illconceived process for comparison. However, with any management tool, process mapping can be taken too far. Sometimes organizations get so carried away with documenting that the flowcharts become too complicated. In flowcharting, people want to know what happens $95 \%$ of the time. The other $5 \%$ rarely happens, so documenting for every instance should be left out (Babicz, 2000). When process mapping reaches all-time confusion levels, "analysis to paralysis" can take over (Adams, 2000).

\section{Conclusions}

Process mapping consists of the tools that enable firms to document, analyze, improve, streamline, and redesign the way their company performs work. Armed with a thorough understanding of the inputs, outputs and interrelationships of each process, analysts can understand how processes interact in their firm's business system, locate process flaws, evaluate which activities add value to customers, streamline and improve work flows, and identify processes that need to be reengineered.

There are many documentation tools available to the accounting student, however processmapping techniques are excellent tools for clearly displaying process flows across organizational boundaries. Recognizing the oftentimes silo nature of the academe, AIS instructors should make sure students are educated regarding the impact of business processes across functional boundaries. Used in conjunction with traditional documentation tools, process-mapping techniques will enable students to document business processes in an attempt to both understand and improve them. In cases where it is important to communicate the "business" relationship that different departments of an organization employ when performing specific business processes, process maps are superior. They very easily and clearly illustrate the nature of processes that require the integration of many different roles throughout the firm.

Process mapping can also be used to create the "could be" state via process redesign. Defining the "could be" state requires "out-of-the-box" thinking and is what true reengineering is all about. To add value to clients, the AIS student should be trained to question the status quo, or "as is" state, of organizational processes. Questioning the status quo of business processes may ultimately lead to the 
improvement of these processes and an improvement in the associated internal controls. The value to the student will be a more marketable skill set, which should include generating new ideas for organizational processes and understanding how to exploit information technology in ways that will enable new organizational possibilities.

\section{References}

1. $\quad$ Adams, L. (2000). "Mapping Yields Manufacturing Insights." Quality Magazine. 39, 5. 62-66.

2. $\quad$ Babicz, G. "Give Your Process the Right Flow." Quality Magazine. 39, 13, 34-36.

3. Boehringer, R. and King, P. Orion Development Group (2000). Process Mapping Gives Great Direction. Dean Hill Systems Limited. Available at:

www.proquis.com/allclear/articles/promap.pdf

4. Hammer M. and Champy, J. (1993). Reengineering the Corporation ( $1^{\text {st }}$ Edition). New York, NY: HarperCollins.

5. Public Accounting Report (1998). "Big Six Dominate Systems Integration Market." July 31, p. 4.

\section{Notes}


Notes 